\title{
Corela
}

Cognition, représentation, langage

HS-7 | 2010

Espace, Préposition, Cognition

\section{Drôle de drupe}

\section{Pierre Cadiot}

\section{OpenEdition}

\section{Journals}

Édition électronique

URL : http://journals.openedition.org/corela/977

DOI : $10.4000 /$ corela. 977

ISSN : 1638-573X

\section{Éditeur}

Cercle linguistique du Centre et de l'Ouest - CerLICO

\section{Référence électronique}

Pierre Cadiot, «Drôle de drupe », Corela [En ligne], HS-7 | 2010, mis en ligne le 31 mai 2010, consulté le 01 mai 2019. URL : http://journals.openedition.org/corela/977 ; DOI : 10.4000/corela.977

Ce document a été généré automatiquement le 1 mai 2019.

\section{(c)}

Corela - cognition, représentation, langage est mis à disposition selon les termes de la licence Creative Commons Attribution - Pas d'Utilisation Commerciale - Partage dans les Mêmes Conditions 4.0 International. 


\title{
Drôle de drupe
}

\author{
Pierre Cadiot
}

1 Sous ce titre, je rends un hommage personnel à Claude Vandeloise. Un jour lointain de juillet - 1995 ou 1996 - nous avons bien ri ensemble en feuilletant les premières pages du "Dictionnaire portatif de la langue françoise, extrait du grand dictionnaire de Pierre Richelet », nouvelle édition refondue par M. De Wailly (1784, Liège) :

Abricot, subst.m. Fruit fort connu.

2 Et comme c'était la saison, nous en avons profité le soir même pour faire une petite orgie d'abricots. Quand l'abricot est en fleur, jours et nuits ont même longueur (dicton français). Mais l'histoire ne pouvait s'arrêter là. Il fallait suivre le devenir lexicographique et encyclopédique du mot et des objets qu'il désigne ${ }^{1}$. C'est ce dont je m'acquitte dans le texte qu'on va lire, et qui voudrait peut-être surtout distraire le lecteur.

\section{Un mini-parcours dictionnairique (dictionnaires généralistes)}

$3 \mathrm{Au}$ delà du Richelet, j'ai consulté quatre dictionnaires qui couvrent les $20^{\text {ème }}$ et $21^{\text {ème }}$ siècles :

Larousse classique illustré, $18^{\text {ème }}$ édition, librairie Larousse, Paris, 1916

abricot, n.m. fruit à noyau dont la chair et la peau tirent sur le jaune, et qui est produit par l'abricotier : compote, confitures d'abricots.

Dictionnaire alphabétique et analogique de la langue française, par Paul Robert, Société du Nouveau Littré, Paris, 1965

abricot, $n$. $m$. (du portug. Albricoque, d'après l'arabe al-barqoûq, le fruit précoce, du lat. praecox ou praecoquus). Fruit de l'abricotier, (drupe) qui se consomme frais, cuit, en compote, confiture, gelée, marmelade, pâte, ou séché par déshydratation, huiles, essences.

Les amandes de l'abricot sont recherchées par les confiseurs.

Le Robert de poche, Langue française\& noms propres, Dictionnaires Le Robert, Paris, 1995 
abricot, n.m. fruit comestible à noyau, à chair et peau jaune orangée.

Wikipedia, 2009

\begin{abstract}
Abricot :c'est un fruit charnu, une drupe, de forme arrondie, possédant un noyau dur contenant une seule grosse graine, ou amande. La chair est sucrée, juteuse, orangée et ferme. La teneur en carotène ou provitamine A est élevée, c'est elle qui donne la couleur orangée et l'abricot est riche en pectines, qui se gonflent facilement d'eau et qui lui confèrent son côté moelleux. La peau, dont la couleur peut aller du jaune au rouge, est parfois piquetée de «taches de rousseur » et se mange. La couleur rouge n'est pas gage de maturité et l'abricot ne mûrit plus après sa cueillette.
\end{abstract}

8 Les progrès en effet sont sensibles par rapport au bon Richelet! Notons qu'il y a trois critères définitoires principaux :

- l'appartenance à la classe des drupes (drupe : « Botanique. Fruit charnu, indéhiscent, renfermant un seul noyau. L'abricot, la pêche, la cerise, la prune, sont des drupes ») (d'après le TLFi))

- la couleur' ${ }^{2}$ l'aspect, la texture;

- la consommation, la comestibilité. ${ }^{3}$

9 Le Robert de poche synthétise ces trois critères en une formule brève et efficace. On est surpris que le Dictionnaire alphabétique et analogique de la langue française ne mentionne ni le noyau ni la couleur. Serait-ce que dans les années soixante, seule importait la consommation?

Plus botaniquement, on peut apprendre ici et là que l'abricot est le fruit d'un arbre, généralement de petite taille, appelé abricotier, de la famille des rosacées, ou encore que son nom scientifique est « Prunus armeniaca L ». Comme tous les pruniers, il relève du sous-genre des Prunophora.

11 Enfin, les dictionnaires à tendance encyclopédique, comme déjà en leur temps Furetière et Trévise, distinguaient plusieurs sortes d'abricots : les abricots ordinaires, qui ne mûrissent qu'à la mi-Juillet; les abricots hâtifs, qui se mangent dès le commencement du même mois; et ceux qu'on nomme petit(s) abricot(s), qui viennent aussi à la mi-Juillet.

S'il est vrai que ce fruit est fort connu - et depuis longtemps en France, depuis le XV'ème siècle au moins - ce que révèlent les variations des trois critères mis en œuvre, c'est que, malgré la facile objectivation de ce nom concret, qu'on dirait, volontiers, mais bien imprudemment, "monosémique», on ne saurait parler de propriétés détachées ou constituées, mais plutôt de caractéristiques d'accès, dont la prégnance est modulable dans le temps et les visées propres de chaque dictionnaire. Nous avons parlé à ce propos de propriétés extrinsèques (Cadiot \& Nemo 1997) simplement pour insister sur cette sémiotisation nécessaire de toute approche définitoire. Des champs expérientiels différents (la perception immédiate, les pratiques culturelles de consommation, les premières synthèses des sciences botaniques, les glissements et appuis " analogiques ", etc.) fournissent des horizons thématiques singuliers (Cadiot et Visetti 2001) auxquels s'adosse le travail de définition. ${ }^{4}$

13 Notons aussi que ces dictionnaires ont ici à décrire un objet particulièrement facile à identifier, qu'il faut cependant inscrire dans des horizons sémiotiques. Pourtant on voit qu'à travers ces rubriques, le mot «abricot », est négligé : on ne nous parle que du fruit, de la drupe. Voyons donc les dictionnaires historiques (des mots). 


\section{Dans les dictionnaires historiques et étymologiques}

14

Albert Dauzat, Dictionnaire étymologique, Larousse-Paris, $11^{\mathrm{ème}}$ édition, 1938

abricot (XVIe s., aubercot, Thenaud, puis abricot) empr. au portugais albricoque, tiré lui-même de l'arabe al-barqoûq qui venait, (article al à part) par l'intermédiaire du grec, du latin praecox ou praecoquus, précoce pour désigner une pêche précoce. dér.: abricotier (1553, Belon); abricoté tranche d'abricot (XVIIe s., Furetière) et abricotin (XVIIIe s. Encycl.) n'ont pas vécu ; abricotine (marbre, XIXe s.).

15 Le Robert, Dictionnaire historique de la langue française, 1992, Dictonnaire Le Robert, 1992.

abricot $\mathrm{n}$. m. est un emprunt indirect ( $\mathrm{XVI}_{\text {ème }}$ siècle) à l'arabe 'al barqūq, lui-même emprunté au grec. Une série de mots apparentés dans les langues romanes (catalan, espagnol, portugais, italien, français) atteste l'histoire compliquée de ce terme, reflet de celle du fruit qu'il désigne. Originaire de Chine, l'abricot a commencé sa carrière méditerranéenne en Syrie. Les Grecs l'avaient appelé armeniakon «fruit d'Arménie " parce que l'Arménie était sa provenance immédiate. Pour les latins, la prunia armeniaca ("prune d'Arménie») 5 se nommait aussi praecoquum «le fruit précoce [...] », mot passé en grec tardif sous la forme praikokion. C'est ce dernier mot grec qui a été adopté par les Arabes, qui cultivèrent mieux que d'autres le fruit, et c'est le mot arabe 'al barqūq où al est l'article et barqūq représente le mot grec praikokion, qui a été adapté dans la péninsule ibérique (le mot espagnol albaricoque est attesté en 1330), puis plus au Nord, et notamment en France.

En français donc, on emploie depuis le $\mathrm{XVI}_{\text {ème }}$ siècle abricot et aubercot; ce dernier vient du catalan albercoc de même origine : c'est le premier qui s'est imposé. En fonction de nom (1740) et d'adjectif, abricot s'applique à une couleur jaune orangé.

Wikipédia, 2009

abricot : Italien albercocca, albicocca; espagnol albaricoque; portugais albricoque. Ce mot français vient de l'espagnol, l'espagnol vient de l'arabe birkouk, et, avec l'article, al birkouk; l'arabe vient du bas-grec qui vient du latin praecoquum, nom donné à l'abricot à cause de sa précocité ; enfin praecoquus n'est pas autre chose qu'une forme de praecox.

On notera selon les périodes et les auteurs, quelques variantes dans les langues romanes modernes :

- Italien : albicócco, albercocca, albicocca

- Provençal : aubricot, ambricot, albricot

- Français : aubercot, arbricot, abricot ${ }^{6}$

mais régulièrement, Catalan albercoc; Espagnol albaricoque; Portugais albricoque.

\section{Histoire (des mots et des choses)}

L'abricot a beaucoup voyagé ${ }^{7}$ : vivant à l'état sauvage de l'Iran à la Mandchourie, il est cultivé depuis 4000 ans dans le Nord-Est de la Chine ${ }^{8}$ (près de la frontière avec la Russie). Il parvient en Arménie après avoir traversé l'Asie centrale. Puis, Grèce. Les Romains l'importèrent de Grèce et l'introduisirent « en Europe » vers 70 avant Jésus-Christ. Puis, petit tour par la Syrie et sans doute, l'Arabie, etc., d'où il revient sérieusement ravaudé (avec notamment l'assimilation de l'article arabe). Beaucoup plus tard, Les Britanniques l'apportèrent dans leurs colonies du continent américain. Et c'est comme ça que pour 
nous, abricot entre dans des phrases où lui sont associés aussi bien les fruits cultivés en France : pommes, poires, pêches, raisins, que les fruits exotiques : figues, dattes, grenades.

\section{Syntagmes plus ou moins figés}

- abricot mûr (J.-K. Huysmans, En route, 1895, p. 80)

- abricot frais (H. De Montherlant, Les Olympiques, 1924, p. 328)

- abricot sec (A. Gide, Journal, 1910, p. 291)

- abricot-pêche (J.-H. Bernardin de Saint-Pierre, Harmonies de la nature, 1814, p. 75)

- noyau d'abricot (F. Mauriac, Le Mystère Frontenac, 1933, p. 43)

- oreillons et oreilles d'abricot (passim)

- parfum d'abricot (Colette, La Maison de Claudine, 1922, p. 7).

Préparations en cuisine ou dans l'industrie alimentaire :

- compote d'abricot (A. Gide, Le Retour du Tchad, 1928, p. 891)

- confiture d'abricot(s) (Ch. Du Bos, Journal, t. 3, 1927, p. 160)

- marmelade d'abricot (Ch. Du Bos, Journal, t. 4, 1928, p. 12)

- gelée d'abricot

- pâte d'abricot (C. Farrère, L'Homme qui assassina, 1907, p. 83)

- tarte à l'abricot (M. Proust, À la recherche du temps perdu, À l'ombre des jeunes filles en fleurs, 1918, p. 904)

- cake, tartelette... d'abricot(s)

- jus d'abricot(s)

- nectar d'abricot

- essence d'abricot

- coulis d'abricots

- sirop d'abricot/abricots au sirop

- mousse d'abricot

- abricots secs

- abricots confits

- huile de noyau d'abricot ${ }^{9}$

- liqueur d'abricots

- pressé d'abricot.

\section{Dérivés largement désuets}

abricoté, abricotée, abricoter, abricotier, abricotin, abricotine, abricotis.

\section{Orthographe et prononciation (d'après TLFi, rubrique 'abricot')}

1. La forme graphique moderne apparaît pour la première fois dans les dictionnaires dans (Cotgrave 1611). Au XVIe siècle, elle coexiste avec la forme aubercot. À partir du XVIIe siècle (Furetière 1690), la forme moderne l'emporte définitivement. 
24 a) Dans la forme disparue aubercot, la deuxième syllabe du mot s'explique par l'emprunt au catalan. Dans la première syllabe, il y a eu vocalisation de [l] devant [b] suivant la loi générale (Bourciez 1967. § 188).

b). L'évolution de albricoque ou albaricoque appelle deux explications : disparition de [1]. Dans un premier stade, assimilation de [l] à [r], d'où arbricot. Une deuxième étape a pu être la dissimilation de ce [r] nouveau par le [r] primitif (cf. Grammont 1950, p. 294) : deux liquides différentes séparées par une occlusive ou une spirante. La première liquide s'assimile à la seconde, puis suivant les parlers, elle subsiste telle quelle ou bien elle évolue sans disparaître, ou bien elle est dissimilée à zéro par la deuxième liquide. Présence de $[\mathrm{t}]$ à la finale. Cas de dissimilation des deux $[\mathrm{k}]$ dont le deuxième est remplacé par une autre occlusive sourde [t]. Peut-être d'abord dans abricotier, '-tier' étant une finale très répandue (cf. '-ier'), on notera l'existence d'un certain nombre de mots (il est vrai de formation plus récente) en '-otier' désignant des arbres (bergamotier, cocotier, indigotier). Timbre de [o] : La plupart des dictionnaires des XIXe et XXe s. transcrivent cette voyelle en finale absolue par [o] fermé suivant la tendance générale de la langue (Fouché, Traité de prononciation française 1959, p. 53). Pourtant Nodier (1847) transcrit Abrico sans circonflexe (comparer bateau Ba-tô, brûlot Brû-lô, etc.). Littré commente ainsi sa transcription $a$-bri-ko : ,... au pluriel $a$-bri-kô ou $a$-bri-ko, la prononciation varie, les uns gardant au pluriel la prononciation du singulier où l'[o] est bref ou ouvert, les autres allongeant $\mathrm{l}^{\prime}$ [o] suivant la règle qui est que $\mathrm{l}^{\prime}[\mathrm{s}]$ du pluriel rend la voyelle longue ou fermée".

XVIe siècle : Oranger, aubercotz, cassiers (Thenaud, 1525). « Ne pouvant sortir par la porte, elles sont contraintes de se jeter par la fenestre, pour aller dans quelque délicieux jardin manger des abricots » (Lanoue, 1587).

XVIIe siècle : Fruit participant de la pêche et de la prune. (...). Il est un peu rouge et jaune en mûrissant. (Furetière. 1690).

XVIIIe siècle : Sorte de fruit à noyau, dont le goût tient de la pêche et de la prune, et dont la chair et la peau tirent sur le jaune (...). Compote d'abricots.. Ac. 1740. Rem. 1.

\section{Variétés}

Parmi les nombreuses variétés existantes, on peut citer les plus couramment produites en France, par ordre d'arrivée sur les étals :

- Lambertin du Languedoc-Roussillon et Orangered de Provence

- Jumbocot rouge du Roussillon et Polonais ou Orangé de Provence

- Bergeron de Rhône-Alpes

- Abricot-pêche de Nancy (ou polonais).

\section{Utilisation, consommation}

On consomme l'abricot frais, mais aussi séché (abricot sec) ou préparé de diverses façons : compote, confiture, gelée, tarte, glace, sorbet, clafoutis, charlotte, crumble, mousse, mirliton, purée, sirop, ratafia (etc.), dans des préparations sucrées (telle le crumble romarin à la compotée d'abricots et au mousseux de citron), parfois combiné (par exemple avec du chocolat, de la vanille, des amandes, des pistaches, etc.), ainsi que dans des plats salés, comme certains tajines, l'abricot farci (ou simplement accompagné) de fromage de chèvre ou 
de brebis, le filet mignon de porc aux abricots, le râble de lapin aux abricots secs ou le lapin aux abricots et aux panais (une recette anglaise). Dans certains pays, comme le Pakistan, on consomme également l'amande située dans le noyau de l'abricot. Dans les variétés commercialisées dans les pays occidentaux, cette amande est consommée en huile (huile d'abricot) et entre dans la composition du persipan en Europe du nord (à la différence du marzipan, dans lequel figurent des amandes) et des fameux biscuits amaretti en Italie. Une eau-de-vie d'abricots s'élabore dans le centre du Valais : elle porte le nom d' abricotine. L'abricot ne doit pas être choisi trop vert car il ne mûrit plus après sa récolte. On emploie les fruits et leur amande pour des pratiques tant médicinales qu'alimentaires ou magiques. Les médecins arabes prescrivaient l'huile extraite des noyaux d'abricot pour soigner les hémorroïdes, les maux de nez et d'oreille. Dans la pratique populaire on l'utilise bien mûr en masque de beauté reconstituant, nourrissant et adoucissant.

\section{Pour la santé}

31 L'abricot, cette "palourde des vergers $»^{10}$, est l'un des fruits les plus intéressants en phytothérapie. Ses vertus sont multiples. Il contient du phosphore et du magnésium, qui nourrissent les cellules du cerveau. C'est un tonifiant intellectuel reconnu depuis longtemps. Il fortifie le sang, favorise la multiplication des globules rouges. L'abricot est donc un atout pour combattre l'anémie. Il est riche en bêta-carotènes qui sont transformés en vitamine A par l'organisme. Cette vitamine A permet d'améliorer la vision nocturne et de protéger la peau. C'est un excellent anti-oxydant qui permettra de retarder les effets du vieillissement et de prévenir l'apparition de certains cancers. Selon une étude récente, il a été prouvé que la consommation quotidienne de 200 grammes d'abricot freinait l'oxydation de certaines structures de l'organisme.

\section{Valeur nutritive}

Abricot cru (valeur nutritive pour $100 \mathrm{~g}$ )

- eau : $86,35 \mathrm{~g}$; cendres totales : $0,75 \mathrm{~g}$; fibres : $2,0 \mathrm{~g}$; valeur énergétique : $48 \mathrm{kcal}$

- protéines : $1,40 \mathrm{~g}$ lipides : 0,39 g ; glucides : 11,12 g sucres simples : 9,24 g

- sels minéraux \& oligo-éléments.

- potassium : $259 \mathrm{mg}$; phosphore : $23 \mathrm{mg}$; calcium : $13 \mathrm{mg}$; magnésium : $10 \mathrm{mg}$

- sodium : $1 \mathrm{mg}$; fer : $390 \mu \mathrm{g}$; zinc : $200 \mu \mathrm{g}$; cuivre : $78 \mu \mathrm{g}$.

- vitamines :

- vitamine $\mathrm{C}: 10 \mathrm{mg}$ vitamine $\mathrm{B} 1: 30 \mu \mathrm{g}$; vitamine B2 : $40 \mu \mathrm{g}$; vitamine B3 : $600 \mu \mathrm{g}$

- vitamine B5 : $240 \mu \mathrm{g}$ vitamine B6 : $54 \mu \mathrm{g}$; vitamine B9 : $0 \mu \mathrm{g}$; vitamine B12 : $0 \mu \mathrm{g}$

- vitamine A : 1926 UI rétinol : $0 \mu \mathrm{g}$; vitamine $\mathrm{E}: 0,89 \mu \mathrm{g}$; vitamine $\mathrm{K}: 3,3 \mu \mathrm{g}$.

- acides gras saturés : $27 \mathrm{mg}$ mono-insaturés : $170 \mathrm{mg}$. ; poly-insaturés : $77 \mathrm{mg}$. ; cholestérol : 0 mg. 


\section{Des fleurs de la meilleure littérature : délices et déchéance}

Et surtout, dans les grands plats vermeils ou dans les jarres d'osier, des fruits, des masses de fruits, figues, dattes, pistaches, jujubes, grenades, abricots, énormes grappes de raisin, plus longues que celles qui firent ployer les épaules des fourriers hébreux dans le pays de Chanaan, lourdes pastèques ouvertes en deux, à la chair humide et rose, avec leurs régimes de grains noirs. (P. Benoît, L'Atlantide, 1919, p. 136).

Voici tous les fruits du verger et la profusion de la corbeille, toutes ces variétés de délices, tout ce qui est né pour fondre, la poire et la pomme dans notre bouche qui réalisent toutes les promesses de la chair, la pêche, l'abricot, la prune profonde, le grapillon aigrelet de la groseille, les raisins bleus et blancs . (P. Claudel, Le Poète regarde la croix, 1938, p. 239.)

C'était elle, c'étaient ses bras, c'était son cou, qui donnaient à ses fruits cette vie amoureuse, cette tiédeur satinée de femme. Sur le banc de vente, à côté, une vieille marchande, une ivrognesse affreuse, n'étalait que des pommes ridées, des poires pendantes comme des seins vides, des abricots cadavéreux, d'un jaune infâme de sorcière. Mais, elle, faisait de son étalage une grande volupté nue. (E. Zola, Le Ventre de Paris, 1873, p. 823.)

\section{La couleur}

ar métonymie (si l'on veut), et à partir du XIX ${ }^{e}$ siècle seulement, sur le cercle des tonalités, le mot abricot correspond d'assez près à la zone intermédiaire « jaune-orangé », voire « orange rosé doux » de l'abricot.

Soit ces quelques exemples :

Et Gilliatt avait entendu cette Parisienne raconter en ces termes ses malheurs : « je suis très ennuyée, je viens de recevoir des gouttes de pluie sur mon chapeau, il est abricot, et c'est une couleur qui ne pardonne pas ».

V. Hugo, Les Travailleurs de la mer, 1866, p. 123.

Des maisons multicolores, abricot, citron, cédrat, qui luisent parmi les oliviers, fruits merveilleux, dans le feuillage ... La vision italienne est une sensualité; les yeux jouissent des couleurs, comme la langue d'un fruit juteux et parfumé.

R. Rolland, Jean-Christophe, La Nouvelle journée, 1912, p. 1444.

(Une) tunique abricot, un chapeau abricot, une robe abricot, un teint abricot

L.-P. Fargue, Le Piéton de Paris, 1939, p. 66.

Selon le TLFi, cet emploi est redoublé par les tournures couleur abricot (A. Gide, Le Retour du Tchad, 1928, p. 1001), couleur de l'abricot (A. T'sertevens, Itinéraire espagnol, 1933, p. 224) ou couleur d'abricot permettant une qualification nuancée : couleur d'abricot mûr, sec (A. Gide, Journal, 1939, p. 291), pâle, frais (H. de Montherland, Les Olympiques, 1924, p. 328). La couleur est « toujours sentie comme celle du fruit».

Le mot se prête bien sûr à des usages littéraires qui en élargissent la valeur. Il a pu évoquer des visions ensoleillées (J. Giono, Colline, 1929, p. 139 : « on peut regarder le soleil; il est rond et roux comme un abricot»), suggérer une idée de gourmandise (nombreuses recettes dans l'Encyclopédie de Diderot). Qualifiant un teint doré, il a été employé comme 
sobriquet d'un homme hâlé (R. Marin du Gard, Les Thibault, La Belle saison, 1922, p. 847-849).

Autant d'exemples illustrant l'idée qu'en tant que terme de couleur, abricot (et ses différentes variantes) ne renvoie pas essentiellement (dans certains emplois seulement) à une zone spécifiée du spectre lumineux, où d'ailleurs, comme on l'a dit, il aurait tendance à parasiter d'autres dénominations dites directes, ou indirectes (orange, orangé, jauneorangé, voire cinabre, vermillon, vermeil). On est dans le registre acatégoriel de l'évocation concrète et synesthésique. A l'inverse, orange/orangé, en dépit de son origine évidemment motivée (le fruit), est par convention une dénomination ayant trouvé sa place comme couleur secondaire, ou « intermédiaire ", créée à partir des deux couleurs primaires du jaune et du rouge (mais aussi associé à la notion de couleur chaude, et dit-on parfois, de couleur de la communication, synonyme d'optimisme, de plaisir et de créativité).

41 Il n'en va pas de même d'abricot, comme de bien d'autres noms et/ou adjectifs de couleur qui sont «donnés par analogie avec des référents matériels, concrets [environnement, milieu naturel] ${ }^{11}$ : [ciel] ciel, bleu azuré, horizon; etc. [végétaux], [fleurs] barbeau, coquelicot, lin, myosotis; etc. [fruits] abricot, cerise, citron, marron, pêche; etc., [métaux, pierres, généralement précieuses] améthyste, ardoise, bronze, émeraude, saphir, smaragdin, turquoise ; etc. [animaux] (bleu, vert-) canard, (gris) souris, serin, etc. ; [matières tinctoriales] bleu de Prusse, indigo, pastel, etc.; [corps humain] chair, incarnat, etc.; [produits fabriqués] beurre frais, bordeaux, champagne, bleu charrette/charron, faïnce, gauloise, gitanes, (bleu-, rose-) layette; [œuvre artistique] Chardin, Nattier, etc.; [races, ethnies] bleu touareg, mordoré, nègre, tête de maure, etc. ; [époque] vert empire ou vert impérial, etc. ; [vêtements] (propres à une fonction) bleu gendarme, bleu roi, brun ramoneur, carmélite, marine, minime, etc. ; propres à un personnage réel ou fictif) céladon, isabelle, etc. »

Même si, comme le disent les spécialistes (notamment A. Mollard-Defour et M. Pastoureau), la couleur nommée, même catégorielle, «joue un rôle toujours plus important que la couleur perçue car elle est chargée d'un pouvoir sémantique, symbolique, affectif ou onirique beaucoup plus fort» (M. Pastoureau, préface in A. Mollard-Defour, 1998 , p. 11), la polysémie et la synesthésie perceptuelle et symbolique des désignations indirectes (elles sont engagées dans la description et l'évocation bien plus que dans la classification) est bien plus prononcée: dans le cas d'abricot, c'est l'ensemble des caractéristiques expérientielles du fruit qui est concrètement sollicité : la fraîcheur ${ }^{12}$, la luminosité, l'humide, le velouté du grain, le pulpe, le charnu, le tacheté (une plage colorée irrégulière, mouchetée de rouge ou piquetée de taches de rousseur, sera facilement dite abricot), mais sans doute aussi une certaine douceur, la sensualité, la santé. Bref, la couleur abricot assume bel et bien, mieux en tous cas que orange, l'essentiel, sinon réellement la totalité, du fruit et des expériences qu'on en a et qu'on en attend. Cette sensualité démultipliée de la couleur est décrite par F. Ponge :

La couleur abricot, qui d'abord nous contacte, après s'être massée en abondance heureuse et bouclée dans la forme du fruit, s'y trouve par miracle en tout point de la pulpe aussi fort que la saveur soutenue.

43 N.B. : Pour ces mêmes raisons fondamentalement, l'abricot trouve aussi bien sûr sa place dans les paradigmes courants des "goûts» ou des "saveurs». Plus que d'autres indicateurs de couleur et de goût, l'abricot tire sa force évocatrice et expressive d'une sorte de resserrement synesthésique. Ainsi de ce vin de Chablis (in Marianne 92, Septembre 2009) présenté comme suit : 
Chablis 2005 plein de verve et de fruit, vif au nez, frais en bouche et doté d'une généreuse finale tirant sur l'abricot et le noyau de pêche.

L'abricot lui-même n'est-il pas " plein de verve et de fruit », « vif » et « frais »?

\section{Un drupe équivoque: le sexe féminin}

$\mathrm{Si}$ « équivoque » évoque bien praecoquus, al-barqoûq, albricoque, il s'agit désormais d'autre chose, dimension incontournable, qu'on introduira par cette citation :

Saviez vous que l'abricot a été introduit en France au $15_{\text {ème }}$ siècle ? Fruit réputé pour sa vitamine A, il fait bénéficier votre corps d'agents anti-oxydants. La vitamine A est une source de jouvence pour notre organisme, car elle permet aux cellules de se renouveler... L'abricot évoque aussi l'érotisme, car en argot l'abricot désigne le sexe de la femme. Sans doute, car il a l'image d'un fruit doux et velouté, fendu et défendu! Découvrez donc une recette délicieuse avec un fruit équivoque rehaussé de cardamome, épiceaphrodisiaque...

[Citation reprise (et partiellement réécrite) d'un paperblog (recette des abricots à la cardamome,2009)]

L'usage idiotique et argotique, bien ancré, d'abricot pour désigner le sexe de la femme n'est pas pour surprendre. Il est même convaincant, linguistiquement réussi, puisque multiplement motivé. Du sexe féminin l'abricot a analogiquement certains caractères plus ou moins sensibles : pulpeux, charnu, tendre, souple, duveteux, fendu, humide. Aussi l'esquisse de lèvres, les variations de l'ovale. D'autres plus ou moins arrangés par l'imaginaire comme la couleur dorée (voire mordorée), voire le sucré.

Dans des représentations en amont, qui anticipent en quelque sorte la dénomination du sexe féminin, la couleur du fruit est associée plus généralement aux promesses de la chair, comme aussi, comme on l'a dit, au teint hâlé des hommes (et des femmes) :

Voici tous les fruits du verger et la profusion de la corbeille, toutes ces variétés de délices, tout ce qui est né pour fondre, la poire et la pomme dans notre bouche qui réalisent toutes les promesses de la chair, la pêche, l'abricot, la prune profonde, le grapillon aigrelet de la groseille, les raisins bleus et blancs ...

P. Claudel, Le Poète regarde la croix, 1938, p. 239.

De rares proverbes pourraient bien jouer leur partition dans cette transition :

Pour abricot mûr, bouche ouverte (proverbe arménien).

Une bouchée de fruit d'immortalité vaut mieux qu'une indigestion d'abricots ( proverbe chinois).

L'abricot est dit-on un "symbole » du sexe féminin ${ }^{13}$, et en argot il est même un symbole dénominatif : à cause de sa forme, de sa texture (l'humide, le velouté...), de son goût même, de ses anticipations tactiles, de ses promesses de bonheur. On admet immédiatement que l'abricot soit « vite devenu une allégorie de la volupté en général et du sexe féminin en particulier » (0. Cair-Hélion, 2007). Quand Nietzsche s'est aventuré à dire du langage qu'il est "cimetière des intuitions ", il ne pensait pas suffisamment à l'abricot.

Dans ces deux extraits, le sexe est crûment désigné :

Quant un moribond l'appelait

Narguant la mort à son chevet

Elle la collait comme un défi

La bouche édentée de l'ancêtre

A son abricot en folie 
Et le papi était guéri

Pierre Perret, La belle fermière, Chansons éroticoquines (2002)

L'as tu vu l'abricot d'la cantinière,

L'as tu vu son p'tit abricot fendu;

Il est noir, noir et blanc,

Il est fendu, par derrière,

Il est noir, noir et blanc,

Il est fendu par devant.

Maurice Fombeure, Soldat (1935).

Francis Ponge est autrement respectueux :

Nulle autre division n'y est d'ailleurs préparée, qu'en deux : c'est un cul d'ange à la renverse, ou d'enfant-jésus sur la nappe,

Et le bran vénitien qui s'amasse en son centre, s'y montre sous le doigt dans la fente

ébauchée.

Francis Ponge, L'abricot, in Pièces $(1999,802)$.

\section{Drôle de pomme d'or}

Nous avons été surpris de trouver dans un texte de l'Eglise du pays de Crussol, cette traduction du Livre des Proverbes:

Des paroles justes dites au bon moment sont comme des abricots d'or avec des ciselures d'argent (Bible, Proverbes 25.11.)

Cette présence inattendue de l'abricot est une exception : on trouve régulièrement pomme d'or dans les traductions françaises de la Bible:

La Sainte Bible, l'Ancien et le Nouveau Testament, par D. Martin, (chez Trenttel et Würtz, libraires, Paris, 1820) :

Telles que sont les pommes d'or émaillées d'argent, telle est la parole dite comme il

faut

La Sainte Bible, Chanoine Crampon, Paris (1960) :

Des pommes d'or sur des ciselures d'argent,

Telle est une parole dite à propos.

Traduction œcuménique (Le Livre de poche, 1979) :

Des pommes d'or avec des motifs d'argent, telle une parole dite à propos

La Bible de Jérusalem (Editions du Cerf, 1998) :

Des pommes d'or avec des ciselures d'argent, telle est une parole dite à propos

La Bible, nouvelle traduction(Bayard, Paris, 2001)

Pommes d'or à feuille d'argent

La parole rondement dite.

Dans l'original hébreu, tapouz (orange) תפוז est une contraction de tapoua'h zahav, «pomme d'or». On ne retrouve pas l'abricot dans les traductions de la Bible auxquelles nous avons eu accès ni en latin, ni en espagnol, ni en italien, ni en anglais, ni en allemand, ni en portugais :

Mala aurea in lectis argenteis qui loquitur verbum in tempore suo.

Manzana de oro con figuras de plata / Es la palabra dicha como conviene.

Come frutti d'oro su vassoio d'argento / così è una parola detta a suo tempo.

Ein Wort geredet zu seiner Zeit, ist wie goldene Äpfel auf silbernen Schalen.

A word fitly spoken is like apples of gold in pictures of silver.

Como maçãs de ouro em salvas de prata, assim é a palavra dita a seu tempo. 
qu' est-ce qu'une pomme d'or? Appelée aussi mini-courge, c'est une courge à la chair fine, fondante et sucrée rappelant le goût de l'artichaut. Les couleurs varient dans la gamme jaune, rouge, orangé.

On sait par ailleurs et surtout que le mot pomme est fortement générique. Il « désigne le fruit comestible d'un arbre en général, qu'il soit à noyau ou à pépins » (Le Robert, Dictionnaire Historique de la Langue Française, rubrique "pomme »). Il y a donc deux versions de la pomme d'or: la courge et le « fruit comestible ». Bien sûr cette dernière version est la bonne!

L'hébreu en fait linguistiquement foi : la pomme d'or de l'Eden est presque sûrement une orange. De même probablement dans la mythologie grecque, celle du jardin des Hespérides. Et pourquoi pas un abricot?

\section{Conclusion}

Fruit, couleur, sexe féminin : ces thématiques, si elles se distinguent nettement, s'organisent aussi dans une continuité sensible. C'est pour faire une juste part à cette sorte de contradiction apparente que nous avons proposé de distinguer entre motif et profil (Cadiot \& Visetti 2001).

Les profils, outre les paradigmes des fruits, de la couleur et de l'anatomie dans sa version argotique, ce sont en l'occurrence certains agencements syntagmatiques comme :

L'abricot est une drupe, un fruit ensoleillé : Tarte à l'abricot

Abricot est une couleur intermédiaire : Un teint abricot

Elle avait l'abricot en folie : Banane Verte Cherche Joli Abricot (Petites Annonces Gratuites Missive).

Les profils ont aussi leur dimension « morpho-syntaxique » :

- Statut substantif vs. adjectif (pour la couleur).

- Genre : masculin pour le fruit et le sexe féminin, mais « neutre » pour la couleur. L'adjectif de couleur, contrairement à plusieurs autres, ne s'accorde pas (cf. * couleur abricote vs. couleur bleue).

- Détermination : détermination d'un « nom concret » pour le fruit et le sexe féminin traités comme tels. Dans le cas du nom de couleur s'y ajoute le déterminant zéro, peut-être lié à une ellipse du substantif 'couleur' ; il tend à être favorisé ${ }^{14}$ :

Cf. :

'Bleu' est une couleur fondamentale

Le 'bleu' est une couleur fondamentale

'Abricot' est une couleur concrète

?l'abricot' est une couleur concrète.

On notera aussi :

Un mur couleur d'abricot

contrastant avec

*Un vin saveur d'abricot.

Le motif, c'est ce germe instable qui associe dans un faisceau synesthésique des expériences sensorielles (couleur, forme, goût, fraîcheur, toucher, ouverture) à des valorisations (saveur, santé, douceur, plaisir, promesse, volupté). 


\section{BIBLIOGRAPHIE}

Brillat-Savarin, J.-A., 1866, Physiologie du goût, Paris, Bureau de la Publication, rue du Coq-Héron

Cadiot, P. \& F.Nemo, 1997, « Propriétés extrinsèques en sémantique lexicale », in Journal of French

Language Studies, $\mathrm{n}^{\circ} 7:$ 1-19

Cadiot, P. \& Y-M. Visetti, 2001, « Motifs, profils, thèmes : une approche globale de la polysémie », in Cahiers de Lexicologie, $79: 5-46$

Cair-Hélion, O., 2007, Les nourritures de la Bible, Allégories et symboles, Paris, Editions du Gerfaut

Cassirer, E. , 1972, La philosophie des formes symboliques, tome 1, Paris, Minuit,

Cassirer, E. , 1977, Substance et Fonction, éléments pour une théorie du concept, Paris, Minuit

Fraser, H., 2004, Constraining abstracness : Phonological representations in the light of color terms, in Cognitive Linguistics 15-3, 239-288

Mollard-Defour, A., 1998, Le dictionnaire des mots et expressions de couleur, Le Rouge, Paris CNRSEditions

Ponge, F. 1999, CEuvres Complètes 1, Bibliothèque de la Pléiade, Paris, Gallimard

Shah, I., 1983, Apprendre à apprendre, Paris, Collection soufisme vivant, Le courrier du livre.

\section{RÉSUMÉS}

Ce texte est une étude longitudinale (notamment dictionnairique) des usages du mot "abricot" en français : le fruit (drupe), la couleur "secondaire", en argot, le sexe féminin, le symbole biblique.C'est aussi une contribution au déploiement de notions comme celles de motif, analogie, figuralité, ainsi qu'un effort pour dépasser les frontières entre lexique, encyclopédie, écriture littéraire et poétique.

The many uses of the word "abricot " in French call for a step by step study which this text endeavours to carry out. It successively reviews the fruit itself (a drupe), the "secondary" colour, its slang use when referring to the vagina and the biblical symbol per se. It aims at being a contribution to the continual revisiting of such notions as lexical drive - « motif » in French analogy, figural process, as well as being an attempt to reconsider the positioning of boundaries between lexicon, encyclopedia, poetic and litarary writing.

\section{INDEX}

Mots-clés : abricot, couleur, mythe, profil, motif

Keywords : apricot, colour, myth, classificatory profile, initial drive 
AUTEUR

PIERRE CADIOT

Université d'Orléans / Laboratoire Ligérien de Linguistque, EA 3850 